\title{
Prediction of Propeller-Induced Hull Pressure Fluctuations via a Potential-Based Method: Study of the Effects of Different Wake Alignment Methods and of the Rudder
}

\author{
Yiran $\mathrm{Su} *$ (1) , Seungnam Kim and Spyros A. Kinnas \\ Ocean Engineering Group, Department of Civil, Architectural and Environmental Engineering, \\ The University of Texas at Austin, Austin, TX 78712, USA; naoestar@utexas.edu (S.K.); \\ kinnas@mail.utexas.edu (S.A.K.) \\ * Correspondence: yiransu@utexas.edu; Tel.: +1-512-706-5771
}

Received: 4 April 2018; Accepted: 2 May 2018; Published: 8 May 2018

\begin{abstract}
In order to predict ship hull pressure fluctuations induced by marine propellers, a combination of several numerical schemes is used. The propeller perturbation flow is solved by the boundary element method (BEM), while the coupling between a BEM solver and a Reynolds-averaged Navier-Stokes (RANS) solver can efficiently predict the effective wake. Based on the BEM solution under the predicted effective wake, the propeller-induced potential on the ship hull can be evaluated. Then, a pressure-BEM solver is used to solve the diffraction pressure on the hull in order to obtain the solid boundary factor which leads to the total hull pressure. This paper briefly introduces the schemes and numerical models. To avoid numerical instability, several simplifications need to be made. The effects of these simplifications are studied, including the rudder effect and the wake alignment model effect.
\end{abstract}

Keywords: hull pressure; pressure fluctuation; diffraction potential; wake alignment scheme; boundary element method; cavitation

\section{Introduction}

Propeller-induced noise and vibration is one of the major issues that threatens onboard comfort, causes mechanical failures, and potentially impacts marine animals. To resolve these issues during the design stage, it is important to have a reliable prediction of the propeller-induced hull pressures. Research has been done on predicting the hull pressure both experimentally and numerically. Most of the experimental studies are performed in the model-scale with multiple pressure transducers mounted on the hull surface above the propeller to monitor the hull pressure [1,2]. According to the level of simplification that can be made, the numerical approaches for underwater noise simulations can be divided into compressible Navier-Stokes equation-based approaches, Lighthill equation-based approaches, Ffowcs-Williams-Hawkings equation-based approaches [3,4], Helmholtz equation-based approaches [5], and hybrids of any two. These equations are usually implemented by either a finite volume method in which the propeller is modelled by a rotating boundary $[4,6]$ or by the boundary element method (BEM) [3,7] in which the propeller is represented by sources and dipoles on the boundary surface.

To numerically predict the propeller-induced hull pressure, the BEM method is often used because the propeller induced pressure field is a small-amplitude high-frequency field which can be decoupled from the background flow. Most of these BEM applications focus on the effect of cavitation because it is the main contributor of high-level noise and vibrations [7,8]. For a cavitating propeller, the cavitation 
source is the major excitation in the acoustic BEM model so that the influence of the propeller lifting force, the blade thickness, and the blade trailing wake can all be neglected. However, the continuous low-level noise and vibration induced by non-cavitating propellers or marginally-cavitating propellers can also be a problem. In this case, the lifting surface, the blade thickness, and the wake all have a comparable influence on the induced pressure.

This paper focuses on a streamlined procedure of predicting the ship hull pressures. A BEM/RANS ${ }^{1}$ interactive scheme is first used to predict the effective wake and the propeller-induced pressure field under this effective wake $[9,10]$. Then, a pressure-BEM solver is used to predict the diffraction pressure which leads to the solid boundary factor. The method considers the lifting surface effect, the blade thickness effect, cavitation source effect, and trailing wake effect so that it can predict the hull pressure induced by either a wetted propeller or a cavitating propeller.

\section{Methodology}

\subsection{Boundary Element Method}

The BEM can be used to solve various types of partial differential equations. In this application, the control Equation (1) can be obtained by inserting the Laplace equation into the Green's third identity. $G$ is Green's function; $S_{B}$ represents all the boundary surfaces in the fluid domain; $n_{q}$ is the normal vector at the point $q$ pointing into the flow field.

$$
\frac{\phi_{p}}{2}=\int_{S_{B}}\left[\frac{\partial \phi_{q}}{\partial n_{q}} G(p, q)-\phi_{q} \frac{\partial G(p, q)}{\partial n_{q}}\right] d s
$$

Equation (1) states that the value of the $\phi_{p}$ at any point on the boundary surface $S_{B}$ depends only on the values of $\phi_{q}$ and $\frac{\partial \phi_{q}}{\partial n_{q}}$ at any point $q$ on the boundary of the body $B$. Moreover, the value of $\phi_{p}$ can be expressed as the superposition of the potentials due to distribution of sources and normal dipoles on the boundary of the body of strengths $\frac{\partial \phi_{q}}{\partial \eta_{q}}$ and $-\phi_{q}$ repectively.

Special care should be given to the branch wake surface $S_{W}$ behind the hydrofoil of propeller. By considering the wake surface, Equation (1) renders:

$$
\frac{\phi_{p}}{2}=\int_{S_{B}}\left[\frac{\partial \phi_{q}}{\partial n_{q}} G(p, q)-\phi_{q} \frac{\partial G(p, q)}{\partial n_{q}}\right] d s-\int_{S_{W}} \Delta \phi_{W} \frac{\partial G(p, q)}{\partial n^{+}} d s
$$

After enforcing the kinematic boundary condition on the strength of the source potential $\frac{\partial \phi_{q}}{\partial n_{q}}$ and the Morino's condition on the wake strength $\Delta \phi_{W}$, Green's foumula finally becomes:

$$
\frac{\phi_{p}}{2}=\int_{S_{B}}\left[\left(-\vec{U}_{i n} \cdot n_{q}\right) G(p, q)-\phi_{q} \frac{\partial G(p, q)}{\partial n_{q}}\right] d s-\int_{S_{W}} \Delta \phi_{W} \frac{\partial G(p, q)}{\partial n^{+}} d s
$$

where $\vec{U}_{\text {in }}$ is the inflow velocity. Equation (3) is a Fredholm integral equation of the second kind for the unknown $\phi$. This analytic formulation will be solved for the unknown quantity, $\phi_{p}$ by using numerical implementation.

To predict the propeller performance, the total flow $\vec{U}_{T}$ can be docomposed to the known inflow $\vec{U}_{I N}$ (or background flow) and the unknown propeller induced flow $\vec{U}_{P}$, which can be treated as a potential flow. Therefore, the propeller perturbation potential is governed by the Laplace equation and can be solved via the boundary element method. In the current boundary element solver, constant strength panels are placed on the surface of the propeller and on the propeller trailing wake surfaces.

1 RANS is the abbreviation of the Reynolds-averaged Navier-Stokes method. 
Equation (3) can then be discretized into a linear matrix system which can be solved by either a direct method or an iterative method.

\subsection{Wake Alignment Model}

Assuming a propeller blade is a lifting body and the blade trailing wake is a material surface on which the potential field is not continuous, the trailing wake surfaces can be treated as a boundary in the BEM model. The strength of the trailing wake is calculated by the Kutta condition and the location of the wake sheet in the downstream is determined by the wake alignment model, which ensures both sides of the wake surface have the same pressure (force free condition).

The basic philosophy behind the wake alignment model is that the wake sheet is a material surface that needs to convect with the local stream. Two types of wake alignment models are available in the current BEM solver depending the time dependency: the steady wake alignment model and the unsteady wake alignment model. Whether the time variation of the incoming flow is considered (unsteady) or not (steady) is the major difference when implementing the alignment procedure. Brief description of each alignment scheme is as follows:

(a) Steady wake alignment: the full wake alignment (FWA) scheme [11,12] considers only the zeroth harmonic of the inflow velocity, therefore the higher harmonic components are neglected in the alignment process. Axisymmetric variation of the incoming flow in radial direction is the most general case, and consequently the propeller performance becomes invariant to the angular position of the blade. The shape of the blade wake is the same for all blades. Figure 1a shows propellers under three different axisymmetric inflows. The induced velocity (or, perturbation velocity) $\vec{U}_{P}$ in Figure $1 \mathrm{~b}$ is evaluated based on the effects from the blade, hub, duct (in case duct geometry is included), and the wake itself. Along with the axisymmetric inflow, the induced velocity constitutes the total velocity prior to implementing the alignment procedure in the FWA.

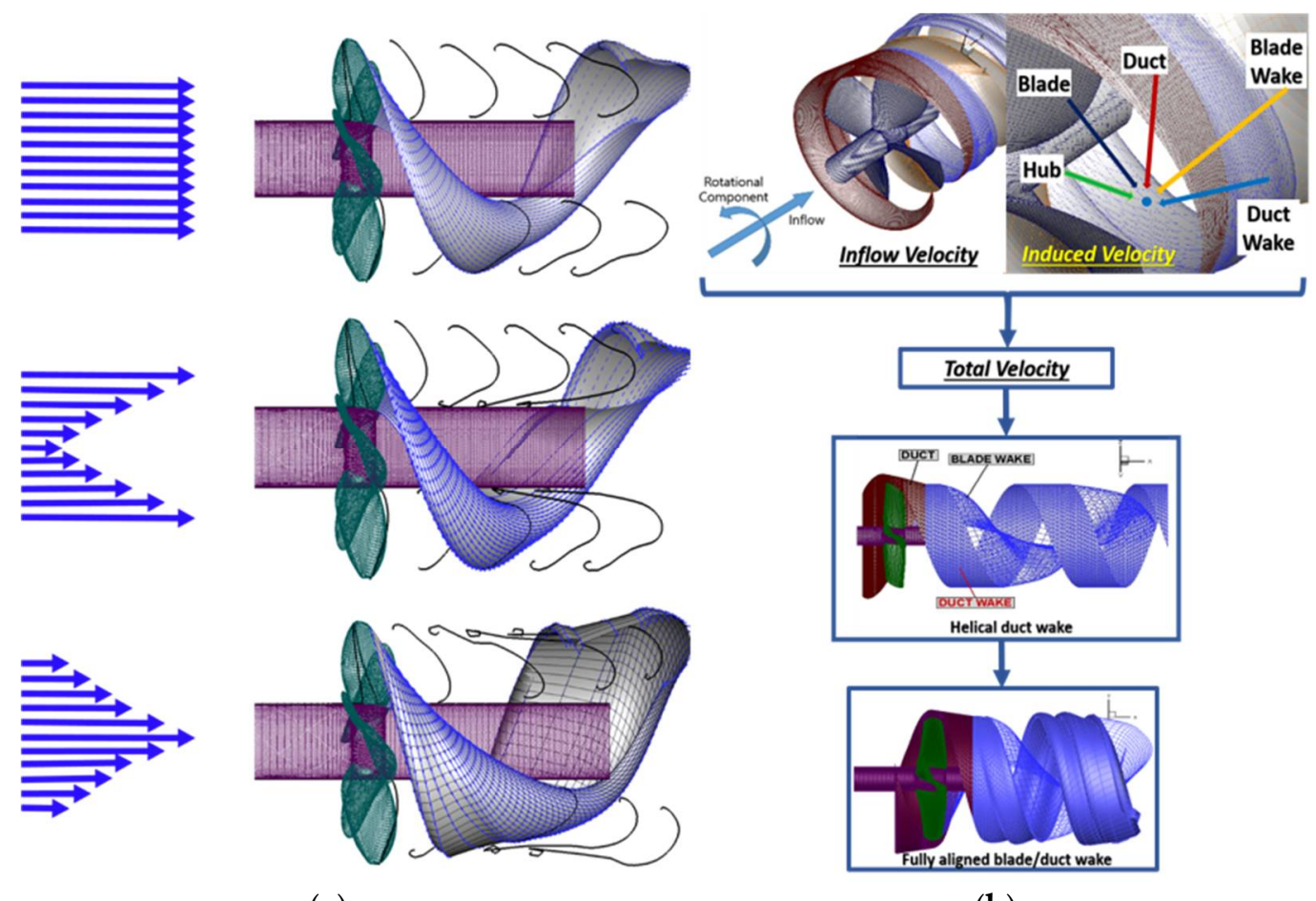

(a)

(b)

Figure 1. (a) Propeller under the axisymmetric inflow; (b) Contributors of the perturbation velocity on the wake surface, taken from Kim [12]. 
(b) Unsteady wake alignment: it is similar to the steady case, but the consideration of time variation of the incoming flow is included. Full harmonics in the inflow are considered to evaluate the velocity components on the wake panels depending on their locations. The alignmen starts with the FWA in steady state $(t=0)$ and procedes into the unsteady alignment model $(t>0)$ using the Euler-explicit sceheme with revolutions in a progressive manner.

$$
\begin{aligned}
X_{i+1}^{n+1} & =X_{i}^{n}+X \vec{U}_{T X_{i}}^{n} \Delta t \\
Y_{i+1}^{n+1} & =Y_{i}^{n}+Y \vec{U}_{T Y_{i}}^{n} \Delta t \\
Z_{i+1}^{n+1} & =Z_{i}^{n}+Z \vec{U}_{T Z_{i}}^{n} \Delta t
\end{aligned}
$$

In the above equations, $i$ and $n$ denote the $i$ th node point at the $n$th time step, $\vec{U}_{T}$ is the total velocity, and $\Delta t$ is the time step size. $X, Y$, and $Z$ are the coordinates of the nodal points on the wake surfaces. Due to the time consideration of the total flow, each blade has a different wake geometry for different blade angles. At each time step, only the key wake is aligned, updated, and then saved before proceeding to the next time step. After that, the saved key wake can be used for other blades when they reach the same blade angle in the future time steps. This procedure will be repeated for several revolutions until the converged unsteady force performances are obtained.

In the current panel model, some elements of the steady wake alignment scheme introduced by Tian \& Kinnas [11] are coupled with the unsteady wake alignment model by Lee [13] to improve the convergence of the alignment procedure. Different from the Lee's unsteady scheme, which evaluates the total velocity at the panel center and then interpolates it into the panel corners, the coupled scheme calculates the total velocity directly at the four corners on each wake panel, as shown in Figure 2. This improves the numerical accuracy and stability. The convergence study on the wake panel numbers showed that the predicted propeller performance was irrelevant to the panel number after 100 panels are used. Detailed description of the unsteady wake alignment model coupled with the FWA can be found in [12].

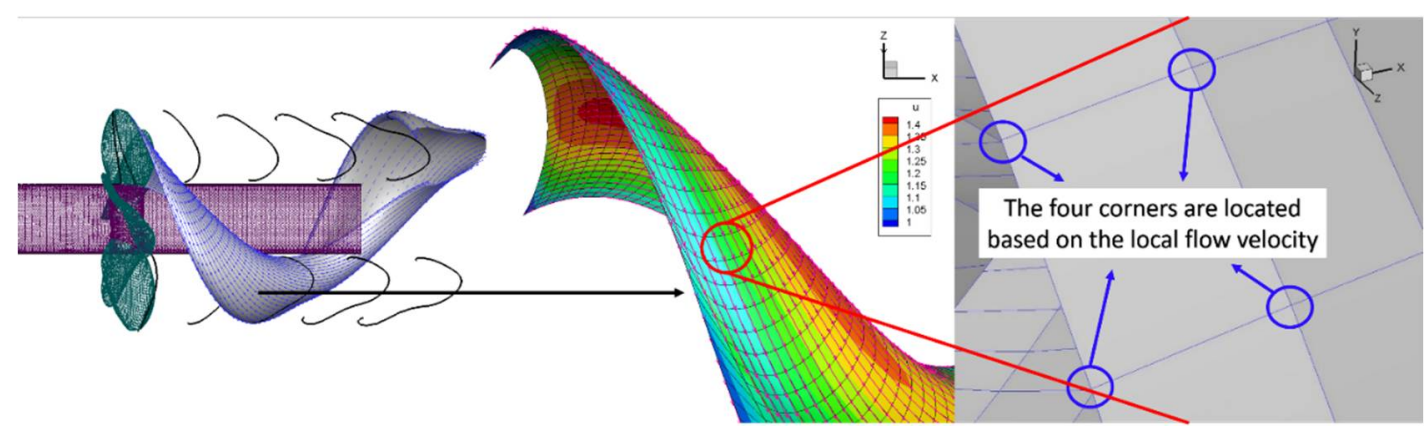

Figure 2. Key wake with the local velocity vectors (red arrows in the middle figure) plotted on each nodal point of the wake, taken from Kim [12].

\subsection{Boundary Element/Reynolds-Averaged Navier-Stokes Interactive Scheme}

In Section 2.1, the BEM solver is used to evaluate the perturbation potential when the inflow $\vec{U}_{I N}$ is given. However, this inflow information is not always available. Some researchers monitor the flow (nominal wake) on the propeller disk plane without the existence of the propeller and then perform an adjustment to the nominal wake to predict the effective wake $\vec{U}_{E F F}$. The effective wake considers the propeller's influence on the flow and can be defined by Equation (5).

$$
\vec{U}_{E F F}=\vec{U}_{T}-\vec{U}_{P}
$$


To predict the effective wake, BEM solver is coupled with a RANS solver, as shown in Figure 3. In the RANS solver, the propeller is represented by a local mass source term which is added to the continuity equation and a local body force term which is added to the momentum equation. The strength of the mass source term and the body force term is determined by the BEM solver. The total flow $\vec{U}_{T}$ is evaluated out of the RANS solver and can be used to calculate the effective wake by Equation (5). The details of this scheme can also be found in the work of Su \& Kinnas [10]. Unlike some other similar implementations, the mass source term is included in the RANS model. It is found that a consistent representation of the blockage effect in both the total flow and the propeller-induced flow is important for the accurate prediction of the effective wake field [10].

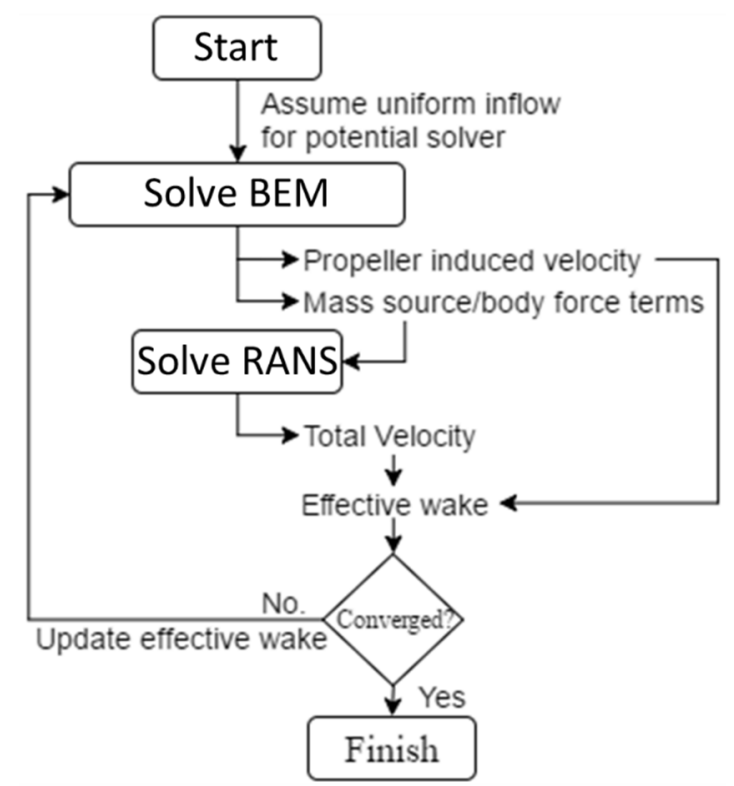

Figure 3. Numerical algorithm of the BEM/RANS ${ }^{2}$ interactive scheme.

\subsection{Boundary Element-Solver for the Oscillating Hull Pressure}

According to the Bernoulli equation, the small amplitude pressure oscillation $P^{(t)}$ can be represented by the steady velocity potential $\Phi^{(t)}$, as shown in Equation (6). In this equation, $\omega$ is the angular velocity of the propeller, $\mathrm{Z}$ is the number of blades, and $\Phi_{n}$ the velocity potential magnitude at a certain frequency.

$$
P^{(t)}=-\rho \frac{\partial \Phi^{(t)}}{\partial t}=-\rho \sum_{n=1,2, \ldots} n Z \omega \Phi_{n} i e^{i n Z \omega t}
$$

Instead of solving the oscillating pressure field, the velocity potential field $\Phi_{n}$ is solved at different frequencies. The potential field $\Phi_{n}$ is governed by the Helmholtz equation. For the near-field small-amplitude pressure fluctuation caused by marine propellers, an infinite sound speed can be assumed so that the Helmholtz equation is reduced to a Laplace equation, as shown in Equation (7).

$$
\nabla^{2} \Phi_{n}=0
$$

Similar to the hydrodynamic BEM, the $n$th total velocity potential field $\Phi_{n}$ can be decomposed to a radiated potential field $\Phi_{n}^{(R)}$ (taken equal to the potential due to the propeller flow in the absence of the

2 BEM is the abbreviation of the boundary element method; RANS is the abbreviation of the Reynolds-averaged Navier-Stokes method. 
hull) and a diffraction potential field $\Phi_{n}^{(D)}$. The radiated potential fields $\Phi_{n}^{(R)}$ is the Fourier series of the unsteady perturbation potential field in the hydrodynamic BEM model which represents the propeller. The lowest frequency for the Fourier decomposition is the blade-passing frequency $Z(\omega / 2 \pi)$.

Based on the boundary element method, the total potential field $\Phi_{n}$ can be solved by Equation (8) where $S_{H}$ is the hull surface and $S_{I}$ is the image of the hull.

$$
\frac{1}{2} \Phi_{n}=\Phi_{n}^{(R)}-\frac{1}{4 \pi} \iint_{S_{H}+S_{I}} \Phi_{n} \frac{\partial G}{\partial n} d S
$$

Different from the hydrodynamic BEM model, the total potential, instead of the diffraction potential, is solved. This eliminates the source-induced potentials term in the boundary integral equation which saves computational time. However, the method cannot be used for the hydrodynamic BEM model because the total velocity field can be vortical and not governed by the Laplace equation. Equation (8) can be solved the same way as in Section 2.1. Constant strength dipole panels are placed on the ship hull and the rudder. The effect from the top tunnel wall is included by an image model. Finally, the unsteady pressure field on the ship hull can be calculated by Equation (8).

\section{Numerical and Experimental Models}

\subsection{Experimental Model}

Numerical schemes are validated by comparing them with the model test results [2,6]. In the experiment, a 1:20 model hull is mounted on the ceiling of a cavitation tunnel, as shown in Figure 4. Eight pressure transducers are placed on the model hull above the propeller. The arrangement and the numbers of each transducer are also shown in Figure 4.

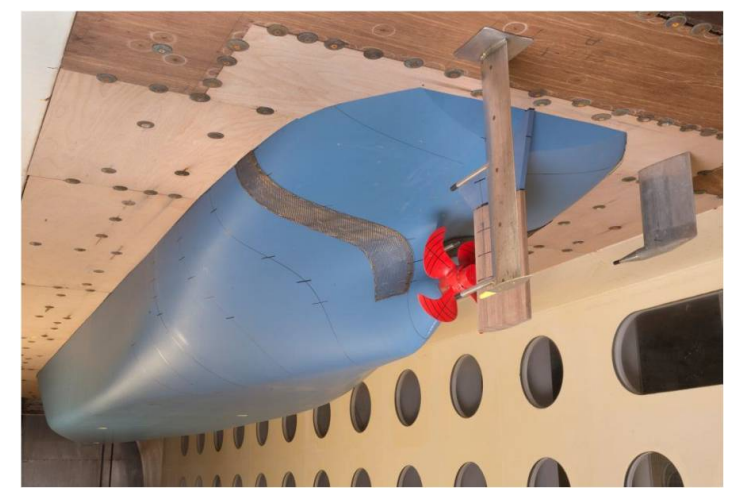

(a)

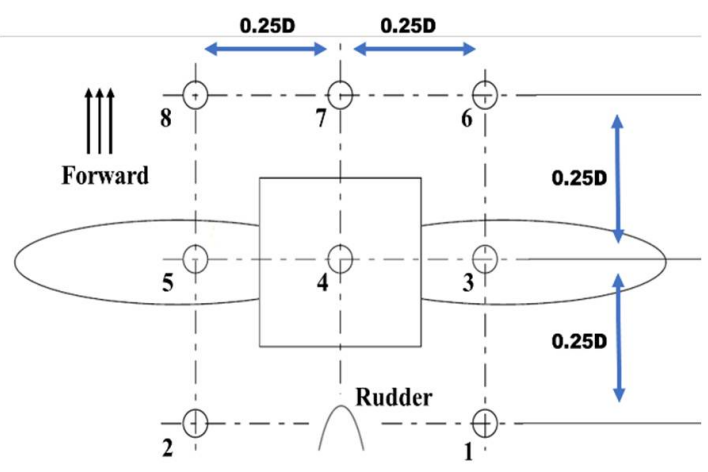

(b)

Figure 4. (a) Photo of the model test facility and (b) pressure transducer arrangement and numbering.

\subsection{Hydrodynamic Model}

In the hydrodynamic model, BEM is coupled with RANS to predict the effective wake as well as the propeller performance. Figure 5 a shows the hydrodynamic BEM model with 80 by 20 panels on a single blade. 4-bladed P2772 propeller [2] is adopted, and the counterclockwise direction about the center of hub is defined to be the negative $x$ direction. Both the steady and the unsteady wake alignment models are tested in fully-wetted flow. Figure 6 shows the RANS model as well as the boundary conditions. The domain size of the RANS model matches the dimensions of the experiment tank. As a result, the effect of the tank is included which can be helpful for reducing the different between the experiment measurement and the numerical prediction. Around 2 million cells are used in this RANS model and the mass/body force zone is shown in Figure $5 b$. 


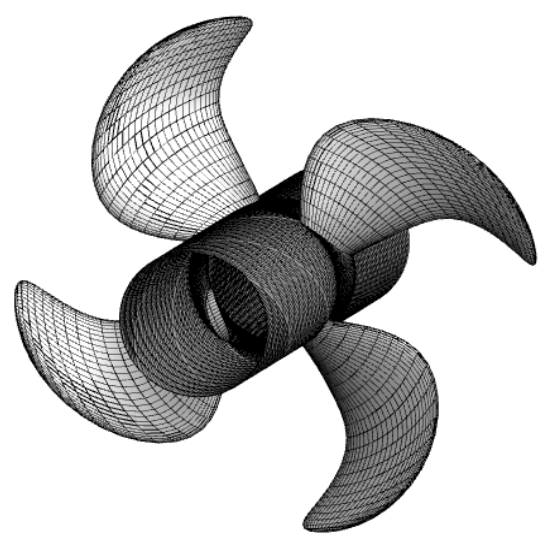

(a)

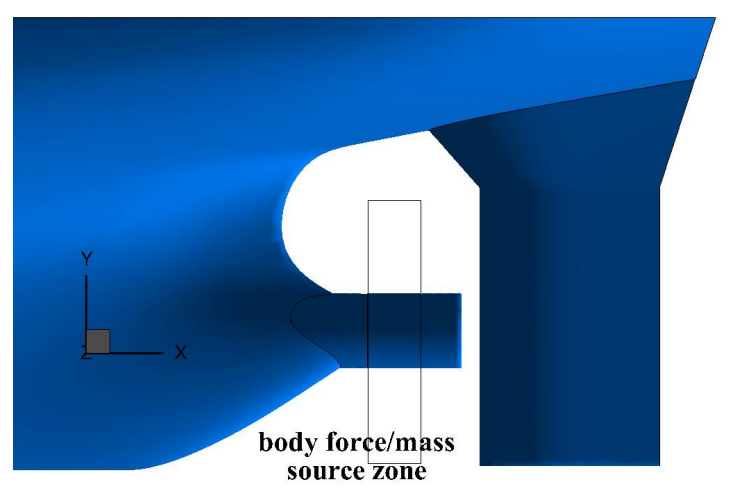

(b)

Figure 5. Propeller geometry generated via (a) BEM and (b) Mass/Body force zone in RANS.

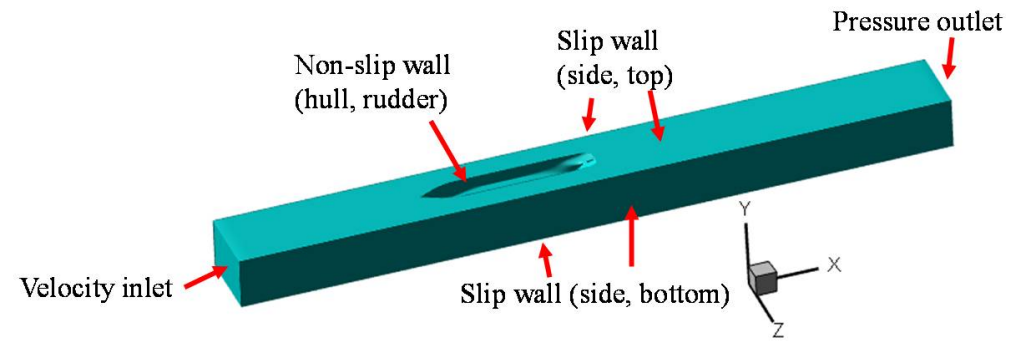

Figure 6. The RANS model in the BEM/RANS scheme.

\subsection{Pressure Boundary Element Model}

In the pressure-BEM model, an external flow problem is solved, which means the BEM panels need to be placed on the surface of a closed body. In reality, since the fore part of the hull has very little influence over the propeller-induced pressure, only the aft part of the hull needs to be included, as shown in Figure 7. An image hull is considered to represent the free surface effect. Therefore, the pressure on the free surface needs not to be solved.

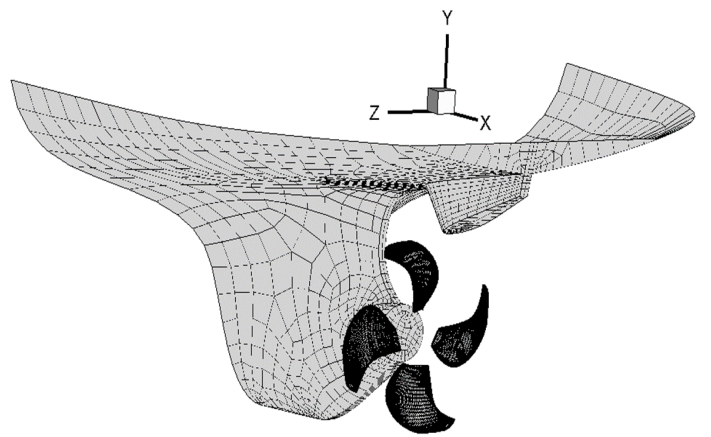

(a)

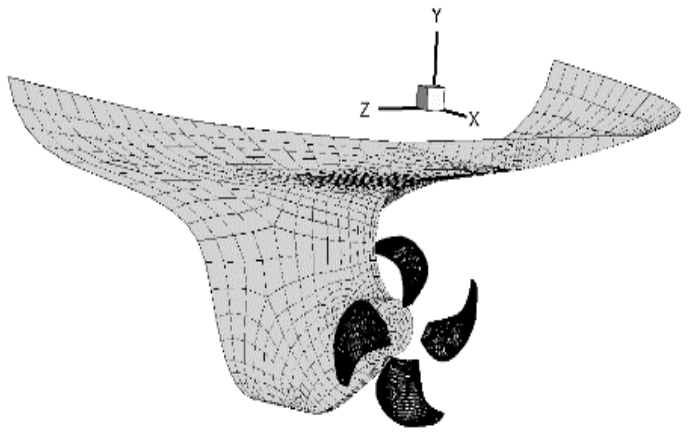

(b)

Figure 7. Pressure-BEM model (a) with and (b) without the upper part of the rudder (the propeller is not a part of this model).

In the current scheme, the wake alignment model does not consider the influence from the rudder. Therefore, it is almost certain that the blade trailing wake penetrates the rudder. Even though the numerical simulation of the trailing wake/rudder interactions was already proposed by He \& Kinnas [14], this modeling is not included in this paper. It is because the interaction between 
the blade trailing wake and the rudder may cause singular radiated pressures on the rudder surface where it touches the edges of the wake panels. To reduce this singular behavior, only the upper part of the rudder is included in the pressure-BEM model. To study the effect of the rudder, a test is also made, in which the rudder is totally neglected in the pressure-BEM model, as shown in Figure 7. Similarly, the length of the hub is reduced so that it does not extend to the propeller zone. It should be noted that the time-averaged interaction of the rudder with the propeller is already included through the presented BEM/RANS algorithm.

Although this rudder geometry is not realistic, it is still used to test and study the validity of the pressure-BEM model in this study. In the future, the hydrodynamic BEM model can be improved so that the wake alignment scheme includes the effect of the rudder. As a result, the full rudder geometry can be used in the pressure-BEM model.

The mesh convergence of the pressure-BEM model has been extensively conducted by Hwang et al. [15]. In that convergence study, a similar mesh density is used on the ship hull. The pressure-BEM model is found to be invariant to the panel numbers used to discretize the hull surface.

\subsection{Unsteady Reynolds-Averaged Navier-Stokes Model}

In addition to the experimental data, unsteady RANS (URANS) simulation is used to provide a second reference. In the URANS model, the hull, the 4-blade propeller, and the rudder are modeled while sliding interfaces are used to handle the motion of the propeller. Around 6 million cells are used in this model and it takes $48 \mathrm{~h}$ on $240 \times 2.7 \mathrm{GHz}$ CPUs to solve this problem in ANSYS Fluent ${ }^{3}$. Eight pressure monitors are placed at the same locations as the pressure transducers in the real experiment.

\section{Results and Comparison with Experiment}

In this paper, all the dimensionless numbers are defined based on the propeller diameter $D$, ship speed $V_{s}$, and the propeller rps $n$. The advance ratio $J_{S}$, the thrust coefficient $K_{T}$, the torque coefficient $K_{Q}$, the cavitation number $\sigma$, and the pressure coefficient $C_{P}$ are defined by the following equations.

$$
\begin{gathered}
J_{S}=\frac{V_{s}}{n D} \\
K_{T}=\frac{T}{\rho n^{2} D^{4}} \\
K_{Q}=\frac{Q}{\rho n^{2} D^{5}} \\
\sigma=\frac{P-P_{V}}{1 / 2 \rho n^{2} D^{2}} \\
C_{P}=\frac{P-P_{\infty}}{1 / 2 \rho n^{2} D^{2}}
\end{gathered}
$$

First, the BEM/RANS interactive scheme is applied to different load conditions by changing advance ratios. The predicted propeller forces are compared to the experimental data, as shown in Figure 8. In the experiment and the BEM/RANS model, the propeller is working behind the ship hull at different advance ratios. A good agreement is obtained between the two. Figure 9 shows the predicted effective wake field for different advance ratios. In Figure 9, the axial effective wake velocity is represented by the gray scale and the in-plane effective wake velocity is plotted by vectors. Since the effective wake is defined at the center of the blade panels, only the mid-chord disk of the 3-dimensional wake field is plotted.

3 The simulation is performed in the Texas Advanced Computing Center (TACC) at The University of Texas at Austin (Austin, TX 78703, USA). URL: http:/ / www.tacc.utexas.edu. 


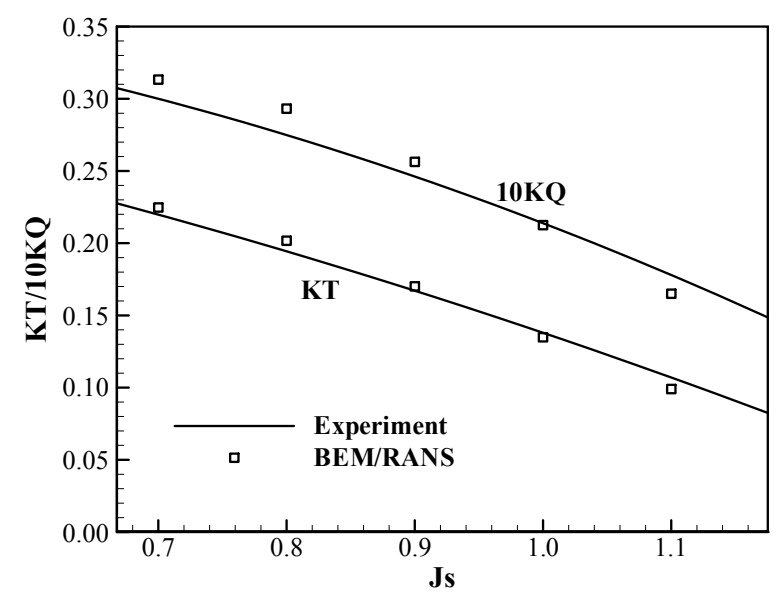

Figure 8. Comparison between the propeller force predicted by the BEM/RANS scheme and that from the experimental data ${ }^{4}$.

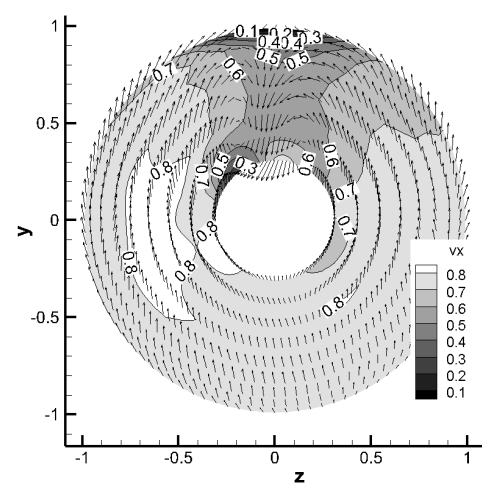

(a)

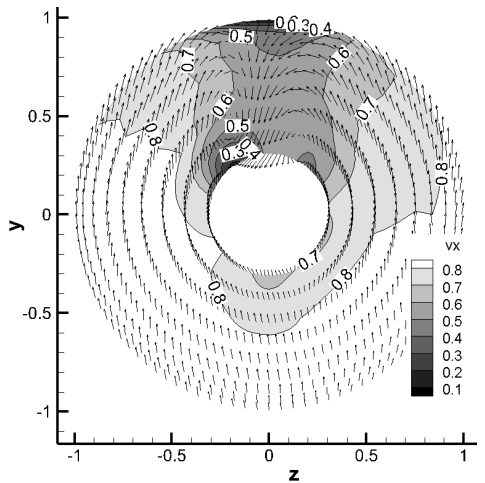

(b)

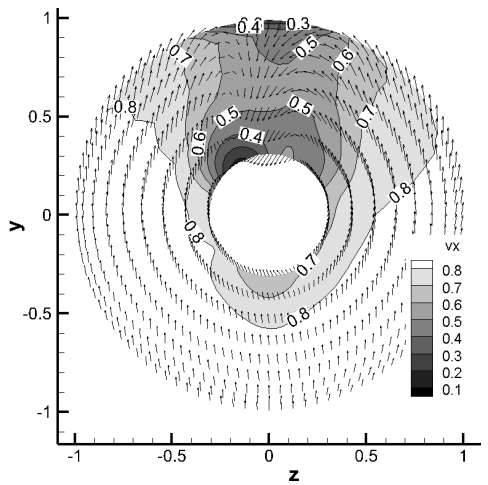

(c)

Figure 9. The predicted effective wake field at the advance ratios of (a) $J s=0.7$; (b) $J s=0.9$; and (c) $J s=1.1$. Only the effective wake distribution on the mid-chord disk is shown. The actual effective wake field may vary in the axial direction.

Then, the same scheme is applied to another load condition in which the advance ratio Js is 0.808 and the cavitation number $\sigma$ is 7.34 . The solution of the BEM/RANS scheme is imported to the pressure-BEM model to evaluate the hull pressure fluctuation on the eight different locations where the pressure transducers are placed. For this load condition, several comparisons are made.

The first comparison study is on the different wake alignment models. Both the steady wake alignment model and the unsteady wake alignment model are tested. In the steady wake alignment scheme, all the non-axisymmetric components of the effective wake are neglected so that the wake geometry, as shown in Figure 10a, does not change with time. More importantly, the positive velocity in the vertical direction of the effective wake, as shown in Figure 9, is neglected. Therefore, the steady wake goes along the axial direction without any inclination. On the other hand, the unsteady wake alignment scheme considers the non-axisymmetric part of the effective wake so that the wake geometry is a function of time (or blade angle), as shown in Figure 10b,c. All the geometries in Figure 10 are plotted in a rotating coordinate which is fixed to the propeller shaft. When the BEM solver is used to predict the propeller mean performance, the difference between the steady and unsteady wake

$4 \mathrm{KT}$ is the abbreviation of thrust coefficient and is defined by Equation (10). KQ is the abbreviation of torque coefficient and is defined by Equation (11). 
alignment model are not significant. However, if the results of the BEM solver are used to evaluate the hull pressure fluctuations, the accurate prediction of the wake geometry becomes more vital.

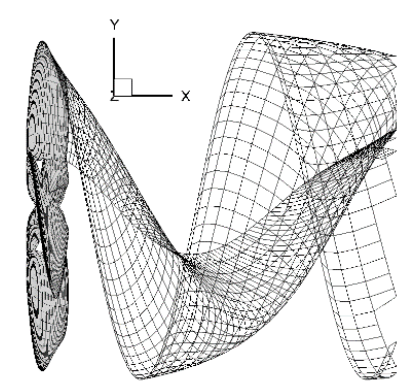

(a)

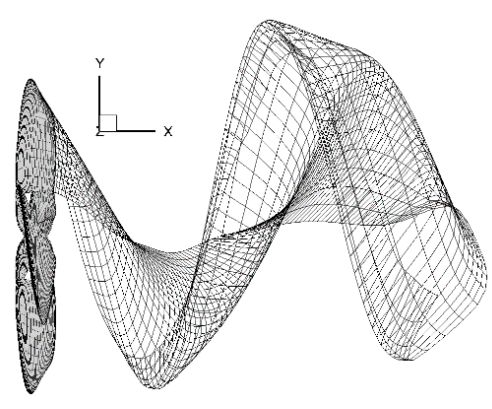

(b)

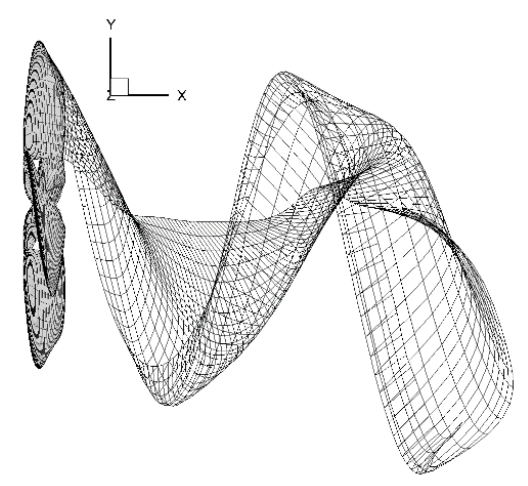

(c)

Figure 10. Comparison of steady wake geometry and unsteady blade wake geometries at different blade angles. The blade-angle ranges between 0 and $2 \pi$ and is defined by the angle the blade has passed starting from the "upright position". The "upright position" means the mid-camber point of the root section is located on the vertical axis above the hub (+y axis). (a) steady wake; (b) unsteady wake 0 degrees; (c) unsteady wake 180 degrees. Please note the wake is shown relative to the propeller fixed system. So the hull is physicallly located above the propeller in (b), but "below" the propeller in (c). Thus, in both cases, (b) and (c), the propeller wake appears to be aligned in a direction parallel to the hull surface.

To study the influence of different wake alignment models, the pressure history monitored by the pressure transducers and by the URANS model are compared with the pressure fluctuation predicted by our scheme with either a steady wake or an unsteady wake. Results are plotted in Figure 11. The unsteady RANS results have a good agreement with the experimental data on transducer T3, T5, T6, and T8. It also correctly predicted the peak pressure pulse amplitude while the location of the peak pressure is shifted towards upstream (see pressure on location T4 and T7). URANS severely under-predicted the pressure pulse at downstream locations (T1 and T2). This can be explained by the numerical diffusion caused by the unstructured mesh. Because the pressure pulses at T1 and T2 highly rely on the behavior of the blade trailing wakes, too much downstream diffusion can smooth out the vortices and diminish the hull pressure pulse.

In terms of the pressure pulses predicted by the pressure-BEM method, the influence of different wake alignment models is not significant for upstream transducer locations (T3-T8), due to their relative long distance to the trailing wake. For downstream points (T1 and T2), however, the difference becomes bigger and unsteady wake alignment improves the results. This is because the non-axisymmetric component of the effective wake pushes the wake towards the hull so that the wake has a stronger influence on the hull pressure.

On transducers T1, T3, T6, T7, and T8, a good agreement is obtained between the BEM-predicted pressure pulses and the experimental data. On transducer T2, the fluctuation amplitude is correctly predicted but the phase angle of the pressure peak is not accurate. This might be attributable to the ignored rudder effect in the unsteady wake alignment model. In other words, although the tip vortex of a blade is close enough to the hull to induce a correct pressure fluctuation amplitude, the time when the tip vortex reaches the closest point to the hull might not be well predicted due to the ignored rudder effect. The complete description of the developed tip vortex cavity in BEM is provided in Lee \& Kinnas [16]. On transducer T4 and T5, current numerical scheme under-predicts the pressure fluctuation amplitudes. Unlike the pressure pulses from the URANS model and from the experimental data, the pressure fluctuation amplitude predicted by the pressure-BEM method is not symmetric about the ship center; the scheme tends to under-predict the pressure pulses on the port 
side. This phenomenon can be traced back to the non-symmetric pattern of the effective wake, as can be seen in Figure 9. As a result, the loading on the blade tip is relatively smaller on the port side and larger on the starboard side.

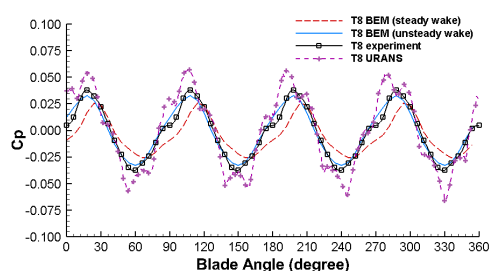

(Transducer 8)

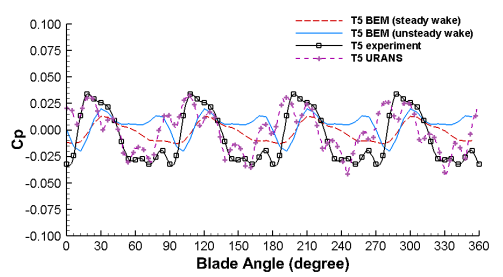

(Transducer 5)

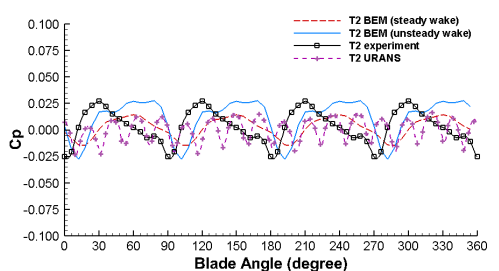

(Transducer 2)

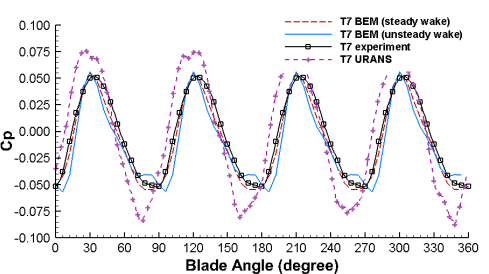

(Transducer 7)

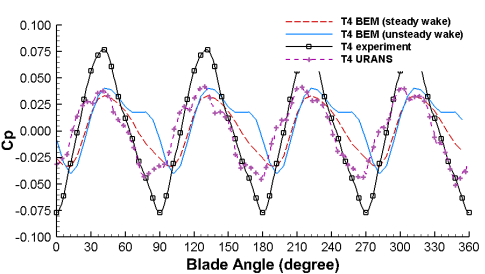

(Transducer 4)

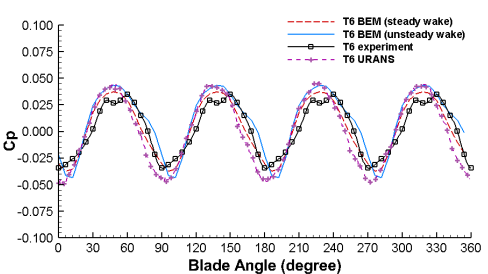

(Transducer 6)

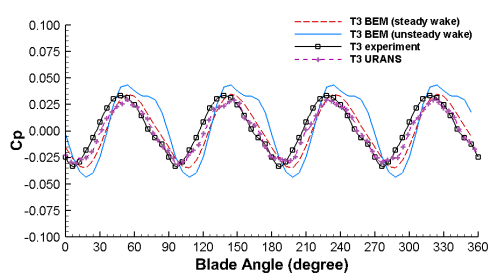

(Transducer 3)

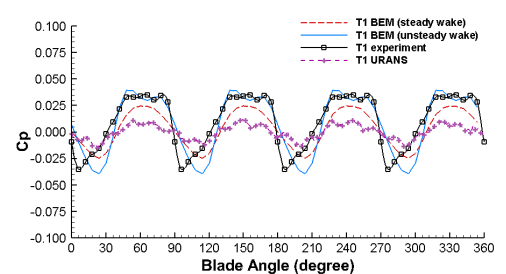

(Transducer 1)

Figure 11. Comparison among the experimental pressure data, Unsteady Reynolds-averaged Navier-Stokes (URANS) method pressure history, and the pressure history predicted by pressure-BEM solver with either a steady or an unsteady wake.

To understand how the hull pressure is affected by the different ways of evaluating the effective wake, another study was made. Two cases were tested in this study and the results are compared with URANS/experiments, as shown in Figure 12. In the first case, the effective wake is evaluated at the center of every BEM panel on the blade. In the second case, the axial-varying effective wake field from the first case is extrapolated to a disk near the leading edge. The disk conforms to the leading edge and has a constant distance ( $2 \%$ of max radius) to the leading edge. The details of both schemes can be found in Tian et al. [9].

Based on Figure 12, the change of locations for evaluating the effective wake can significantly change the predicted hull pressures, especially for downstream monitors. For monitors T1, T2, and T8, defining the effective wake at the center of every blade panel shows a clear advantage over the other method which defines the effective wake at a leading-edge disk. At other monitors (T3 to T7), there is no clear advantage for either of methods. In summary, the hull pressure pulses are shown to be very sensitive to the effective wake field. Although our current axial-varying effective wake shows some advantages, it still needs to be improved due to the inconsistency in the pressure of several monitors between the BEM results and the experimental data. 


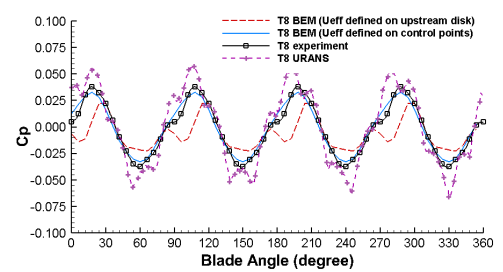

(Transducer 8)

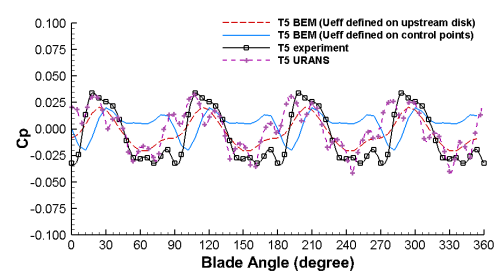

(Transducer 5)

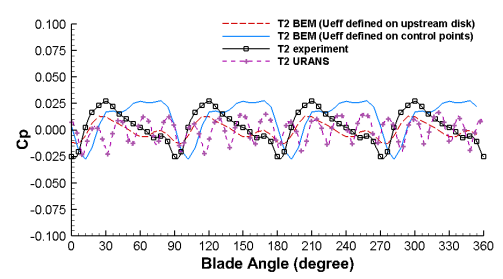

(Transducer 2)

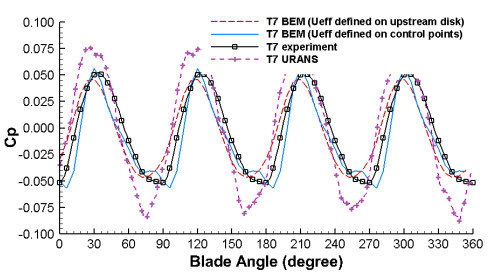

(Transducer 7)

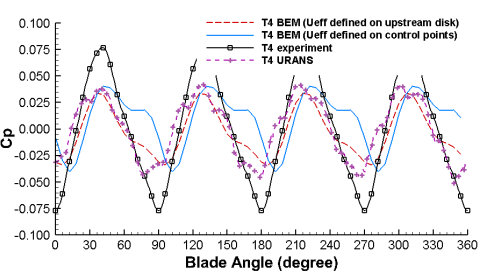

(Transducer 4)

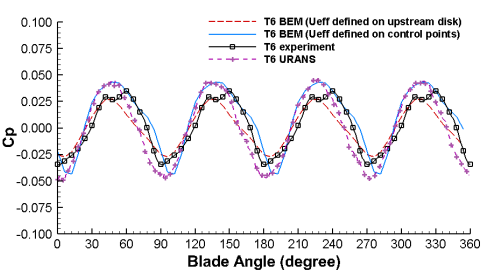

(Transducer 6)

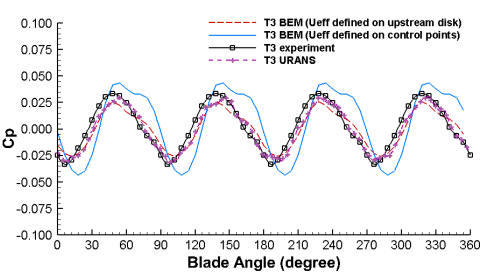

(Transducer 3)

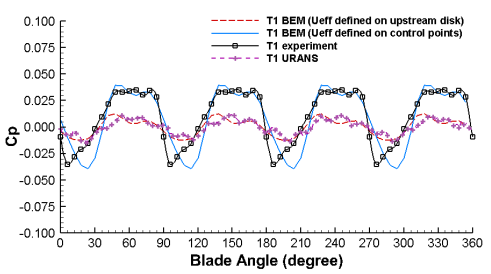

(Transducer 1)

Figure 12. Comparison of hull pressures predicted by the pressure-BEM solver with different treatment of the effective wake field: (a) Effective wake field is evaluated at the center of every blade BEM panel (blue solid line); (b) The effective wake field is extrapolated to the upstream curved surface close to the blade leading edge (red dash line).

The last comparison study focuses on the effect of the rudder. According to Section 3.3, in order to maintain numerical stability, only the upper part of the rudder is used in the pressure-BEM model, as can be seen in Figure 7a. The underlying assumption is that the lower part of the rudder is far from the pressure transducer locations and therefore, has a negligible influence towards the hull pressure fluctuation. To study whether this is a good approximation, we tried two different rudder geometries in the pressure-BEM model: one is the original geometry which is shown in Figure 7a; the other geometry totally neglects the rudder part, as can be seen in Figure $7 \mathrm{~b}$. The predicted hull pressures from both cases are compared in Figure 13. According to the figure, the difference is negligible on T4 and T7 while the pressure on T1 is affective by the rudder geometry. This is because T1 is at a downstream location which is close to the leading edge of the rudder. We can also further claim that by including the upper part of the rudder, only the local pressure field is noticeably affected. This supports our original assumption that the lower part of the rudder would have an even smaller influence on the predicted hull pressures, given its relatively longer distance from the hull.

It is worth noting that a more complete way for solving the numerical stability issue is to include the wake-rudder interaction in both the unsteady wake alignment model and the pressure-BEM model. This can be a future topic of this research.

Another neglected effect is from the blade boundary layer. The boundary layer effect, similar to the blade thickness, can be represented by source panels in the BEM model. The BEM solver can be used together with a boundary integral solver to determine the displacement thickness $[17,18]$. Then, the thickness can be converted into source strength and applied to the BEM solver. The displacement thickness can be as large as $15 \%$ of the blade thickness. Also, the source induced pressure decays slower $\left(\sim R^{-1}\right)$ with distance compared to the dipole induced pressure $\left(\sim R^{-2}\right)$. Therefore, it is possible 
that the boundary layer effect contributes to the downstream hull pressure fluctuation. The effect of boundary layer is another future topic of this research.

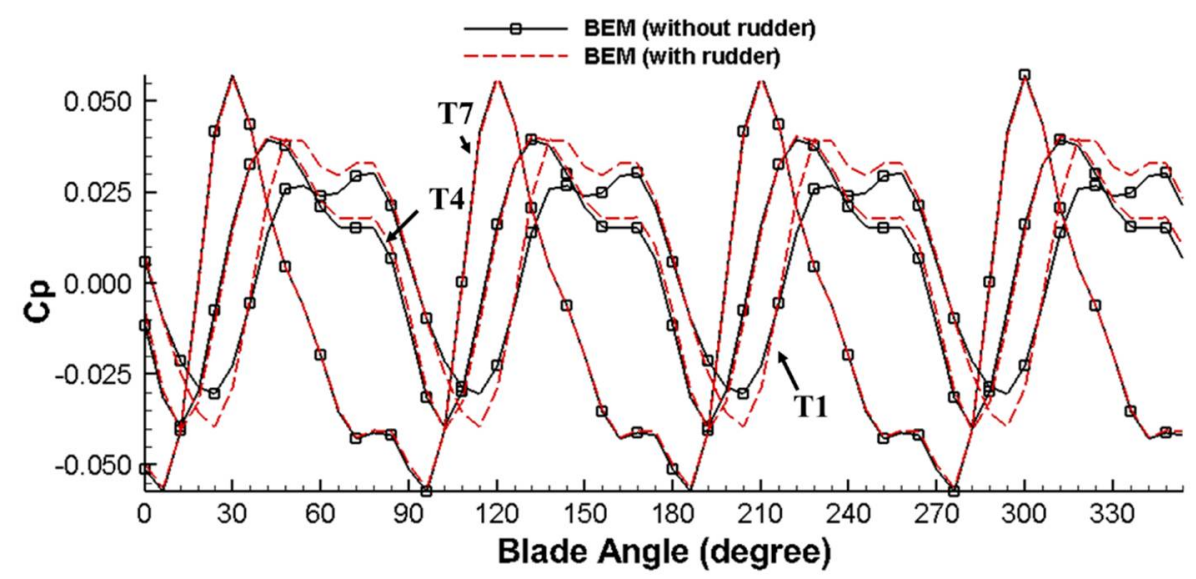

Figure 13. Study of the level of simplification on the rudder geometry in the pressure-BEM model. The blade-angle ranges between 0 and $2 \pi$ and is defined by the angle the blade has passed starting from the "upright position". The "upright position" means the mid-camber point of the root section is located on the vertical axis above the hub (+y axis).

\section{Conclusions and Future Work}

In this paper, several schemes are introduced to predict the propeller-induced hull pressure fluctuations. A BEM/RANS solver is first used to determine the effective wake and the propeller performance under the effective wake. Then, a pressure-BEM model is used to calculate the pressure on the ship hull. In the pressure-BEM model, only the upper part of the rudder is used to avoid singular radiated pressure on the rudder. A comparison study was made which indicated that excluding the lower part of the rudder is a good approximation. The effect of wake alignment model is also studied. The unsteady wake alignment scheme behaves better in terms of predicting the downstream hull pressures. Including the non-axisymmetric effective wake and using the unsteady wake alignment scheme improved the accuracy of the location of the wake and subsequently the accuracy of predicted the hull pressures.

Future work includes studying the blade boundary layer effect on the hull pressure, incorporating the unsteady wake-rudder interaction in the numerical scheme, and studying the effect of a finite speed of sound. The scheme of predicting the effective wake can also be improved in terms of accuracy.

Author Contributions: Y.S. participated in the development and improvement of the BEM pressure prediction model. S.K. participated in the development of different types of wake alignment models. S.A.K. provided guidance throughout the research.

Acknowledgments: Support for this research was provided by the U.S. Office of Naval Research (Grant Nos. N00014-14-1-0303 and N00014-18-1-2276; Ki-Han Kim) partly by Phases VII and VIII of the "Consortium on Cavitation Performance of High Speed Propulsors".

Conflicts of Interest: The authors declare no conflicts of interest.

\section{References}

1. Van Wijngaarden, E. Recent developments in predicting propeller-induced hull pressure pulses. In Proceedings of the 1st International Ship Noise and Vibration Conference, London, UK, 20-21 June 2005.

2. Tani, G.; Viviani, M.; Hallander, J.; Johansson, T.; Rizzuto, E. Propeller underwater radiated noise: A comparison between model scale measurements in two different facilities and full scale measurement. Appl. Ocean Res. 2016, 56, 48-66. [CrossRef] 
3. Salvatore, F.; Testa, C.; Greco, L. Coupled hydrodynamics-hydroacoustics BEM modeling of marine propellers operating in a wakefield. In Proceedings of the First International Symposium on Marine Propulsors (SMP '09), Trondheim, Norway, 22-24 June 2009; pp. 537-547.

4. Kellett, P.; Turan, O.; Incecik, A. A study of numerical ship underwater noise prediction. Ocean Eng. 2013, 66, 113-120. [CrossRef]

5. Seol, H.; Jung, B.; Suh, J.-C.; Lee, S. Prediction of non-cavitating underwater propeller noise. J. Sound Vib. 2002, 257, 131-156. [CrossRef]

6. Li, D.-Q.; Hallander, J.; Johansson, T.; Karlsson, R. Cavitation dynamics and underwater radiated noise signature of a ship with cavitating propeller. In Proceedings of the VI International Conference on Computational Methods in Marine Engineering (MARINE 2015), Rome, Italy, 15-17 June 2015.

7. Lee, K.; Lee, J.; Kim, D.; Kim, K.; Seong, W. Propeller sheet cavitation noise source modeling and inversion. J. Sound Vib. 2014, 333, 1356-1368. [CrossRef]

8. Seol, H.; Suh, J.-C.; Lee, S. Development of hybrid method for the prediction of underwater propeller noise. J. Sound Vib. 2005, 288, 345-360. [CrossRef]

9. Tian, Y.; Jeon, C.H.; Kinnas, S.A. Effective Wake Calculation/Application to Ducted Propellers. J. Ship Res. 2014, 58, 1-13. [CrossRef]

10. Su, Y.; Kinnas, S.A. A Generalized Potential/RANS Interactive Method for the Prediction of Propulsor Performance. J. Ship Res. 2017, 61, 214-229. [CrossRef]

11. Tian, Y.; Kinnas, S.A. A Wake Model for the Prediction of Propeller Performance at Low Advance Ratios. Int. J. Rotating Mach. 2012, 2012, 372364. [CrossRef]

12. Kim, S. An Improved Full Wake Alignment Scheme for the Prediction of Open/Ducted Propeller Performance in Steady and Unsteady Flow. Master's Thesis, Ocean Engineering Group, CAEE, The University of Texas at Austin, Austin, TX, USA, August 2017.

13. Lee, H. Modeling of Unsteady Wake Alignment and Developed Tip Vortex Cavitation. Ph.D. Thesis, Ocean Engineering Group, CAEE, The University of Texas at Austin, Austin, TX, USA, August 2002.

14. He, L.; Kinnas, S.A. Numerical simulation of unsteady propeller/rudder interaction. Int. J. Nav. Archit. Ocean Eng. 2017, 9, 677-692. [CrossRef]

15. Hwang, Y.; Sun, H.; Kinnas, S.A. Prediction of Hull Pressure Fluctuations Induced by Single and Twin Propellers. In Proceedings of the Society of Naval Architects and Marine Engineers Propellers/Shafting 2006 Symposium, Williamsburg, VA, USA, 12-13 September 2006.

16. Lee, H.; Kinnas, S.A. Application of BEM in the Prediction of Unsteady Blade Sheet and Developed Tip Vortex Cavitation on Marine Propellers. J. Ship Res. 2004, 48, 15-30.

17. Drela, M. XFOIL: An analysis and design system for low Reynolds number airfoils. In Low Reynolds Number Aerodynamics; Springer: Berlin/Heidelberg, Germany, 1989; Volume 54.

18. Kinnas, S.A.; Yu, X.; Tian, Y. Prediction of Propeller Performance under High Loading Conditions with Viscous/Inviscid Interaction and a New Wake Alignment Model. In Proceedings of the 29th Symposium on Naval Hydrodynamics, Gothenburg, Sweden, 26-31 August 2012.

(C) 2018 by the authors. Licensee MDPI, Basel, Switzerland. This article is an open access article distributed under the terms and conditions of the Creative Commons Attribution (CC BY) license (http:/ / creativecommons.org/licenses/by/4.0/). 